\title{
Rastrear cáncer colorrectal con fibrosigmoideoscopía subdiagnostica adenomas avanzados proximales
}

Detection of proximal adenomatous polyps with screening sigmoidoscopy: a systematic review and meta-analysis of screening colonoscopy. Lewis JD, Kemmic Ng, Hung KE et al. Arch Intern Med, 2003;163:413-20.

\section{Objetivo}

Determinar la efectividad de la fibrosigmoideoscopía en relación a la colonoscopía como estrategia de rastreo de cáncer colorrectal (CCR).

\section{Fuente y Selección de Datos}

Los autores realizaron un meta-análisis de las publicaciones indexadas en Medline sobre estudios de colonoscopía de rastreo en pacientes asintomáticos. Fueron excluidos lo estudios con pacientes con antecedentes de pólipos o cáncer. El total de estudios incluidos fue de 13. El rango de pacientes incluidos en los estudios fue de 90 a 3121. En seis estudios se incluyeron pacientes con antecedentes familiares de CCR. Se definió como adenoma avanzado a las lesiones con cáncer, displasias de alto grado, componente velloso o tamaño mayor de $10 \mathrm{~mm}$. Se definió como le- siones proximales a aquellas fuera del alcance del endoscopio.

\section{Resultados Principales}

La prevalencia global de adenomas proximales fue de 22,6\% (rango 4,7 a $30,5 \%$ ) y de adenomas avanzados entre el 2,5 y el $7,3 \%$. La prevalencia global de neoplasias proximales aisladas (sin lesiones distales) fue del $16,3 \%$ (IC $95 \% 13,6$ a $19,1 \%$ ) alcanzando un $5 \%$ la prevalencia de adenomas avanzados.

\section{Conclusiones}

Los pólipos distales adenomatosos se asocian a una prevalencia aumentada de neoplasias proximales. Hasta el $5 \%$ de los pacientes a los que se les efectúa una colonoscopía como rastreo de CCR presentan lesiones proximales avanzadas que no habrían sido reconocibles con sigmoideoscopía.

\section{Comentario}

EI CCR es una causa importante de muerte por cáncer. Estimaciones del Ministerio de Salud informan que en Argentina cada año hay 10.300 nuevos casos y más de 5.000 muertes. Esta afección presenta casi todas las características que se requieren para ser pasible de un rastreo poblacional efectivo: alta prevalencia, largo periodo precanceroso (la transformación pólipo-cáncer demora en general 10 años) y tratamiento en estadios tempranos que mejora la sobrevida.

Si bien existen estrategias de comprobada eficacia estas distan de ser simples y aceptadas por la población lo que limita el impacto final. Por ejemplo, en países como EEUU, donde la población recibe educación continua al respecto, sólo una minoría cumple adecuadamente el rastreo y no se ha logrado una reducción marcada de la mortalidad ${ }^{2}$. Sin embargo luego de muchos años de dilema y controversias por falta de evidencia epidemiológica, el rastreo de esta patología esta totalmente aceptado y consensuado en las principales sociedades mundiales.

Actualmente se considera que cualquiera de las siguientes estrategias es adecuada: sangre oculta en materia fecal (SOMF) anual, fibrosigmoideoscopía cada cinco años, combinación de ambos, colon por enema cada cinco años o colonoscopía cada diez años. Si bien de todas estas estrategias solo hay evidencia directa fundamentada en estudios prospectivos controlados para la SOMF${ }^{2}$. La evidencia indirecta recientemente acumulada a favor de la colonoscopía cada diez años, ha llevado en EEUU a que esta estrategia, junto con la SOMF anual, sean las únicas cubiertas por el seguro Medicare.

El presente estudio analiza la capacidad de la sigmoideoscopía en la detección de neoplasia colónicas analizando la asociación entre lesiones a su alcance y aquellas que pueden pasar inad- vertidas sin un estudio completo del colon. Demuestra que uno de cada 15 pacientes con fibrosigmoideoscopía normal presenta una adenoma proximal y uno de cada 42 tiene adenomas avanzados (incluyendo adenocarcinomas). También surge de los datos que los pólipos hiperplásicos no justifican un estudio completo. Sin embargo, conocer la estirpe histológica implica la toma de una biopsia con sus costos y complicaciones. Además, ciertamente, no permite descartar la presencia de una lesión sincrónica encontrada al azar. Como lo comentan los autores, estos datos son argumentos a favor del rastreo con colonoscopía. Mas aún, estudios realizados en el EE.UU demuestran que la colonoscopía cada 10 años es costo-efectiva ${ }^{3}$ (si bien estas conclusiones no son extrapolables a nuestro país)

Para decidir cual es la mejor estrategia debe tenerse en cuenta, además, la disponibilidad y calidad de los estudios implementados (ej. índice de llegada al ciego en las colonoscopías, índice de complicaciones, calidad de los estudios radiológicos) que varían ostensiblemente entre los distintos centros. Debido a la complejidad de este tema, es probable que por muchos años más, las estrategias de rastreo sigan variando y la conducta en poblaciones cautivas deba tomarse teniendo en cuenta los aspectos mencionados así como la decisión final de los pacientes.

Conclusión del comentador: El rastro de CCR exclusivamente con fibrosigmoideoscopía puede dejar sin diagnósstico adenomas avanzados proximales.

\section{Dr. Carlos A. Vaccaro}

[ Director del Programa de Cáncer Hereditario. Sector de Coloprotología. Servicio de Cirugía General. Hospital Italiano de Buenos Aires ]

\section{Referencias}

1. Edwards B. Cancer Statistics, Review 1973-1996. Bethsada, Md: Cancer Institute, 1999.

2. Pignone M, Rich M, Teutsch SM, Berg AO, Lohr KN. Screening for colorectal cancer in adults. Systematic Evidence Review No. 7 (Prepared by the Research Triangle Institute-University of North Carolina Evidence-based Practice Center under Contract No. 290-97-0011). AHRQ Publication No. 02-S003. Rockville, MD: Agency for Healthcare Research and Quality. June 2002. Available on the AHRQ Web site at http://www.ahrq.gov/clinic/serfiles.htm

3. Pignone M, Saha S, Hoerger T, Mandelblatt J. Cost-effectiveness analyses of colorectal cancer screening: a systematic review for the U.S. Preventive Services Task Force. Ann Intern Med. 2002 Jul 16;137(2):96-104. 